\title{
How are fraction values constructed?
}

\author{
Abstract \\ Fractions are a difficult symbolic number format to master for children and adults \\ alike. Errors made with symbolic fraction numbers suggest that even experienced adults \\ can lack fluency with fractions. The importance of the fraction format in formal education \\ has motivated a great deal of research in focused on why fractions are so difficult for \\ peoples to use. In the current study, we investigate the question of how people construct an \\ internal representation of fraction values from the symbols. We present data across two \\ behavioral experiments and mathematical modeling to demonstrate how people construct \\ fraction values from the constituent parts. In the behavioral experiments we manipulate \\ the temporal dynamics of stimulus presentation in the canonical number comparison task. \\ We demonstrate that a small delay in the presentation of specific fraction components \\ affects the relative response time. We find that participants are significantly faster to \\ complete numerator delayed trials, suggesting that participants can use the denominator to \\ begin construction of the fraction value. We further characterize the cognitive processes \\ and strategies that account for the behavioral data using a dynamic systems neural \\ network mathematical model. We conclude that participants begin the construction of \\ fraction values with the denominator and can initiate such construction before the \\ numerator is known.
}


FRACTION VALUES

Learning about fractions, and the symbolic number system, in general, is about learning to construct meaning about magnitudes from symbols. Children must learn how a small set of symbols 0 - 9 can be used in various permutations to symbolize an infinite set of numbers. People commonly have difficulty in using fraction notation (e.g., Braithwaite, Pyke, \& Siegler, 2017; Bright, Behr, Post, \& Wachsmuth, 1988; Mack, 1990). The use of fraction notation is rife with errors for both children and adults. This difficulty may be due to a lack of fluency with the notation and a problem with how people construct values from the symbolic notation. The common errors made with fractions may reveal the cognitive processes people employ in constructing fraction values.

In this study, we evaluate how people construct fraction values from the composite symbols. While there is not currently a comprehensive account of how people construct fraction values, there are some relevant findings. Eye-tracking data suggests that, when comparing fractions, adults initially focus on the denominators of the two fraction values. (Huber, Moeller, \& Nuerk, 2014; Obersteiner \& Tumpek, 2016). Theoretically, why are denominators important? They signify the total number of parts in the whole. We manipulated the task dynamics of the fraction comparison task to more explicitly characterize the cognitive processes involved. Each fraction comparison depends on the relative values conveyed by two numerators and two denominators. We examine how variations in the presentation timing for each component are associated with variations in participants' response time on the fraction comparison task. We vary time as a way to investigate what, if anything, is done with the incomplete information during the delay 
FRACTION VALUES

period. We combine empirical experimentation with mathematical modeling to create an account of how participants combine components to construct fraction values.

What level of characterization is required for an account of fraction value construction? The goal of experimental psychology, prediction of people's behavior across different individuals and contexts, relies on the characterization of the underlying processes that give rise to the behavior. We specify processes as aspects of cognition that may not be directly revealed by behavioral data alone. We differentiate this from 'box and arrow" style mental representation models in that we quantify processes using a mathematical model allowing for more explicit model predictions. For example, consider the A-not-B error, a behavioral phenomenon observed on a simple find the hidden object task. Explanations for this phenomenon have greatly benefited from an approach that evaluated the change in participants' behavior when the timing and spacing of the task stimulus were varied (e.g., Smith 1999). Combining this task dynamics empirical approach with mathematical modeling has resulted in a comprehensive explanation of the behavior, how it varies across contexts and how underlying processes can explain both (e.g., Simmering, Schutte, \& Spencer, 2008; Smith, Thelen, Titzer, \& Mclin, 1999; Spencer, Smith, \& Thelen, 2001).

We investigate how people construct fraction values by examining the effect of stimulus presentation timing on the fraction comparison task. We evaluate the accuracy and speed in comparing fractions. The current approach posits an automated representation of well-known and highly practiced number symbols (i.e., 7, 3), while values for less familiar symbols need to be constructed (i.e., 12/27). We predict that for some incomplete fraction presentations participants will be able to initialize the construction of 
FRACTION VALUES

the fraction value prior to viewing the complete fraction. Based on prior work we predict that the presence of a denominator, even if the numerator is absent, will allow for fraction value construction to begin (Huber et al., 2014; Obersteiner \& Tumpek, 2016).

\section{The Current Study}

Across two experiments we show that the timing of the presentations of symbols can greatly affect the response time of fraction comparison. Each fraction pair presentation contains four components: denominator 1 , numerator 1 , denominator 2 , and numerator 2 . The four components can be presented simultaneously or with a delay for one or more components (see Figure 1). We used three sequences: No Delay, Numerator Delay, and Denominator Delay.

What predictions do accounts of fraction comparison make?

The current Dynamic Fraction account predicts that the relative presentation timing of the fractions and whole numbers will affect the relative timing of fraction comparison due to how the participant constructs the fractions. If the person can initiate fraction value construction during the delay, then response times will be relatively faster than if fraction value construction does not. The dynamic fraction construction account predicts that some information may be used by the person to begin to construct the fraction value even before all components of the fraction are known. To evaluate this prediction, we will compare cases in which the numerator or denominator is withheld during the delay period. 
FRACTION VALUES

Using a formal mathematical model we find that participants' data is consistent with a fraction value construction and comparison process that is initiated when only denominators are available. The model also evaluates two alternatives, (1) fraction construction process that does not initiate until all components are available, (2) the use a shortcut strategy that relies on comparing fraction components but not the actual fraction values. Mathematical modeling results demonstrate that neither of these alternatives matches participants' behavior in experiments 1 and 2 .

\section{Experiment 1}

In this experiment, we sought to provide within subject evidence that delayed numerator trials take less response time than delayed denominator trials. Participants completed the fraction comparison task across three conditions; No Delay, Numerator Delay, and Denominator Delay. The experimental design was approved through the University of Maryland Internal Review Board.

\section{Method}

Participants: Adults $(\mathrm{n}=60)$ were recruited online via Amazon Mechanical Turk (age median 32, minimum 20, maximum 63). The experiment was designed to take approximately 20 minutes. Participants were compensated $\$ 3.00$. We excluded participants who did not indicate they were attending to the task. The median performance was well above $90 \%$ and the median response time under $1.5 \mathrm{~s}$. The following analysis includes all participants whom both scored at least $70 \%$ overall and had no more than $20 \%$ 
FRACTION VALUES

of trials as response time outliers (greater than 6 seconds) for any condition, $n=48,80 \%$ of participants.

Power analysis for a repeated measures ANOVA show that for power of 0.80 and medium effect $(\mathrm{f}=0.15$ ) the minimum sample size is 37 . Extensive pilot testing shows that participant response times for the fraction comparison task across the repeated factors are highly correlated $(r=.80)$.

Procedure: Participants completed a fraction comparison task. Instructions indicated that two fractions would appear on the screen, participants were to indicate which fraction was a larger value via a button press. Participants were instructed that there may be a slight delay in the display of either fraction and they should respond as quickly and accurately as possible once both fractions were fully displayed. Any responses made prior to full fraction display were ignored. Fractions remained on the screen until the participant responded.

Stimuli from this task were based on the stimulus set in prior work (Meert, Grégoire, \& Noël, 2009), see the Appendix for the full list. There were 64 unique comparisons, each presented three times for a total of 196 comparisons for each participant. Fraction comparisons stimuli were blocked by presentation style: No Delay, Numerator Delay, and Denominator Delay. The order of the blocks was randomized, as was the order of the stimuli within each block. For Numerator Delay comparisons, both fractions were initially presented with a blank numerator. The numerator then was displayed after a delay of 2 seconds. For the Denominator Delay comparisons, both fractions were initially presented 
FRACTION VALUES

with blank denominators. For No Delay comparisons, both complete fractions were presented with no delay period.

\section{Results and Discussion}

We analyzed participant reaction time across all presentation styles. For each participant we excluded reaction times longer than 6 seconds, representing less than $2 \%$ of trials. For trials with no delay, the overall mean was $1.70 \mathrm{~s}$, with an overall accuracy of $94 \%$. For trials with a numerator delay, the overall mean was $1.22 \mathrm{~s}$, with an overall accuracy of $95 \%$. For trials with a denominator delay, the overall mean was $1.34 \mathrm{~s}$, with an overall accuracy of $95 \%$.

We evaluated a 2(delay type) X 2(congruency) repeated measures ANOVA. There was a significant main effect for consistency, $F(1,47)=12.76, p<.001, \eta^{2} G=0.004$, and for delay type, $F(1,47)=4.16, p=0.04, \eta^{2} G=0.01$. The interaction between the two was also significant, $F(1,47)=13.03, p<.001, \eta^{2} G=0.01$. We found that numerator delayed trials $(M$ $=1.22 \mathrm{~s})$ were significantly faster than denominator delayed trials $(\mathrm{M}=1.34)$.

The results suggest that participants are faster to compare fractions the numerator is delayed. We also found that participants were faster to compare fractions if the components were congruent with the fraction values, consistent with the Natural Number bias. All fraction comparison pairs in experiment 1 contained common components. It is possible that participants' strategy for comparing fraction values was affected by the inclusion of common components. A potential explanation is that participants rely on a component strategy that does not involve comparing the fraction values. Since all trials in this study contain some common component participants might ignore the common 
FRACTION VALUES

components and compare the natural number values of the non-matching components. For example, for $11 / 15$ vs. $11 / 19$, the participant would simply compare 15 and 19 to determine which fraction is larger. On trials with common denominators, participants would merely compare the numerators. On trials with common numerators, participants would compare the denominators. If participants were consistently using this strategy, there would not be a difference in reaction time between numerator delay and denominator delay trials. If the hypothesis that fraction values are constructed denominator first were correct, then it would be expected that numerator delay fractions would be processed faster than denominator delay fractions.

\section{Experiment 2}

For experiment 2 we repeated the experimental paradigm from Experiment 1 but used a different stimulus set than in experiment 1 . In this experiment, fraction comparisons did not contain common components.

Participants: Adults ( $\mathrm{n}=44)$ were recruited online via Amazon Mechanical Turk (age median 31, minimum 20, maximum 53). The experiment was designed to take approximately 20 minutes. Participants were compensated $\$ 3.00$. We excluded participants who did not indicate they were attending to the task. The median performance was well above $90 \%$ and the median response time under $1.5 \mathrm{~s}$. The analysis includes all participants whom both scored at least $70 \%$ overall and had no more than $20 \%$ of trials as 
FRACTION VALUES

response time outliers (greater than 6 seconds), $\mathrm{n}=35$ or approximately $80 \%$ of participants.

Procedure: Same as Experiment 1.

Fraction Comparison Task: The same as Experiment 1 with the exception of the use of fraction comparisons without common components (see Appendix X for the list from (Obersteiner \& Tumpek, 2016). Additionally, participants viewed only Numerator or Denominator delay presentation styles. The presentation style was blocked with order randomized between participants.

\section{Results and Discussion}

We analyzed participant reaction time across all comparisons. For each participant we excluded reaction times longer than 6 seconds, representing less than $2 \%$ of trials. For trials with a numerator delay, the overall mean was $1.22 \mathrm{~s}$, with an overall accuracy of $92 \%$. For trials with a denominator delay, the overall mean was $1.32 \mathrm{~s}$, with an overall accuracy of 91\%.

We evaluated a 2(delay type) X 2(congruency) repeated measures ANOVA. There was a significant main effect for consistency $F(2,68)=15.54, p<.001, \eta^{2} G=.044$, and for delay type $F(1,34)=4.29, p=0.04, \eta^{2} G=.008$. The interaction between the two was also significant $F(2,68)=6.28 p=.003 \eta^{2} G=.007$. We again found that numerator delayed trials $(M=1.22 \mathrm{~s})$ were faster than denominator delayed trials $(M=1.32)$. 
FRACTION VALUES

\section{General Discussion}

The critical question is what does shorter response times for numerator delayed comparisons tell us about participants' fraction value construction? What processing, if any, are participants engaging in when presented with incomplete information about the fraction comparison? Are participants using the incomplete information to facilitate the comparison once the fractions are complete or are they just waiting for the information?

We conclude that faster response times for numerator delays indicates that participants begin constructing the fraction values when only the denominator is present. Our interpretation that denominators are primary in fraction value construction is consistent with recent evidence from eye-tracking showing denominators are attended to before numerators (Huber et al., 2014; Obersteiner \& Tumpek, 2016).

\section{Alternate explanations}

An alternate hypothesis is that the delay merely gives participants additional time to inhibit the natural number values and there is no difference in which component is delayed. This hypothesis aligns with a construction process in which is agonistic to which component, numerator or denominator, is delayed. Comparison of fractions involves inhibition of the natural number values that correspond to the fraction components (Meert et al. 2009). For example, comparing $3 / 7$ vs. $2 / 3$ followed by 7 vs. 3 is associated with slower comparison reaction time for 7 vs. 3 because the natural number values were inhibited in the prior fraction comparison. If participants were inhibiting natural number values, we would expect a similar response times across both delay conditions. That is not consistent with the current results. 
FRACTION VALUES

To demonstrate how participants' fraction value construction process affects their response time we constructed a mathematical model to evaluate the possible explanations formally. In the following mathematical modeling analysis, we address the potential processes for constructing fraction values: a denominator dependent construction and a component agnostic construction. We also evaluate the possibility that participants are not fully constructing fraction values and use a shortcut based on the common components that make up the fractions. We evaluate how well each model condition matches the current behavioral data in terms of the relative response times for each presentation style.

\section{Mathematical Modeling}

To further characterize the governing dynamics of the fraction comparison we constructed a mathematical model of the task. The purpose of the models is to examine how hypothesized internal representations and their dynamics give rise to the patterns of behavior reported in experiments 1 and 2 . The model is constructed to simulate behavior on the fraction comparison task. The model makes fraction comparisons corresponding to each of the conditions: No Delay, Numerator Delay, and Denominator Delay.

We assume that in processing a fraction, the natural number values are relatively quickly represented while the fraction value is constructed slowly depending on the presentation timing of fraction components. Fraction value construction involves inhibiting the representation of the natural numbers. Thus in the first milliseconds of viewing $7 / 18$, the representation of 7 and 18 is relatively strong, while the representation of $7 / 18$ is 
FRACTION VALUES

relatively weak. The important question is what processes are involved in constructing the fraction value.

We hypothesize that fraction value construction is initiated with the denominator as opposed to the numerator, or with both simultaneously. The model study includes three conditions to evaluate our hypothesis regarding the construction of fraction values:

Model Condition A: construction of fraction values requires all components to be present. Model Condition B: the construction of fraction values begins as soon as the denominator is present.

Model Condition C: construction of fraction values requires all components to be present, but the model will use shortcuts when common components are presented first. (e.g. x/10 vs. $\mathrm{x} / 10$ )

\section{Method}

The model completes a fraction comparison task using the same comparisons as human participants in Experiments 1 and 2. The model input includes numerators and denominators for two fraction values. The model constructs estimates of both fractions and makes a decision about which value is larger. The timing and accuracy of the fraction comparison depend on the change in model values across time steps. The more time steps required in making a comparison correspond to longer reaction times. The input of the fraction components can be delayed across the timesteps in the same manner that components were presented to participants on a delay in the behavioral experiments. In this sense, the model can evaluate fraction comparisons across the same set of conditions: 
FRACTION VALUES

No Delay, Single Numerator Delay, Single Denominator Delay, Double Numerator Delay, Double Denominator Delay, Mixed Delay, and Single Fraction Delay.

All model conditions follow the same general process for completing a fraction comparison. The model output is a decision of which of two values, fraction A and fraction $\mathrm{B}$, is largest. To do that, the model constructs the value of the fractions from the component inputs: Numerators $\mathrm{N}_{\mathrm{A}}, \mathrm{N}_{\mathrm{B}}$ and denominators $\mathrm{D}_{\mathrm{A}}, \mathrm{D}_{\mathrm{B}}$. There are two avenues of comparison in the model architecture (Figure 7). One avenue uses of $\mathrm{N}_{\mathrm{A}}$ and $\mathrm{D}_{\mathrm{A}}$ to construct the fraction $A$ value, $\mathrm{N}_{B}$ and $\mathrm{D}_{\mathrm{B}}$ to construct the fraction $\mathrm{B}$ value and then directly compares fractions $\mathrm{A}$ and $\mathrm{B}$ to make the decision. The second avenue compares the natural number parts of the fractions, numerator to numerator and denominator to denominator. Thus it is possible for the natural number components to interfere with the fraction value decision, giving rise to a Natural Number bias effect. The inputs layer representations corresponding to numerators and denominators are the same that has previously been used in natural number comparison (Prather, 2014).

We evaluate three separate versions of the mathematical model that correspond to different methods of fraction value construction and strategy use. We evaluate the number of time-steps taken in completing each comparison. The model completed the same comparison trials as participants. Each model condition is structured to make a decision in comparing two fraction values. Model input is the four fraction components, numerator A, denominator A, numerator B and denominator B. The model takes those input layers and combines them to construct a representation of the fraction values for fraction $\mathrm{A}$ and fraction B. Those two values then feed into a decision layer. Activation from the layers for 
FRACTION VALUES

fraction A and fraction B compete with each other; the decision layer output reflects the timing and result of that competition.

Model condition A: all components. This model condition simply waits until all components are available to construct the fraction value. Fraction component delays are implemented by setting relevant input vectors to zero for the duration of the delay (100 time-steps). For example, for the Single Numerator Delay condition one numerator input vector is set to zero for the first 100 time points. After 100 time steps the numerator "appears" and the vector is set to the relevant value. For this condition, we also set any other layers related to the delayed component to zero for the delay duration. Thus a delay of Numerator 1 also corresponds to a delay of the Fraction Value vector and the fraction comparison vector.

Condition B: denominator primary. When a fraction component is delayed fraction value proceeds as soon as the denominator is present. Thus for a trial in which the numerator is delayed, but the denominator is not, the fraction value construction is not delayed. Delays are implemented by setting relevant input vectors to zero for the duration of the delay (100 timesteps). If the denominator is the component delayed, then other layers for fraction value and fraction comparison will also be set to zero during the delay. If the denominator is present, while the numerator is delayed, then the fraction value and fraction comparison layers are not set the zero. 
FRACTION VALUES

Condition C: shortcut strategy. This condition is the same as condition A with the exception that the model will employ the common components strategy. For each comparison, if the common components are delayed the model simply compares the noncommon components to begin to decide before both fractions are presented. For example, if 6/x and 5/x are initially displayed, and we assume each fraction comparison has some common components then we know that $6 / x$ must be greater than $5 / x$. This strategy also works for $\mathrm{x} / 5$ vs. $\mathrm{x} / 6$ except that in this case, $\mathrm{x} / 5$ is larger. Construction of fraction values requires all components to be present, but the model will use shortcuts if common components are presented first. (e.g. x/10 vs x/10). Model condition C is meant to address the possibility that the behavioral data is due to strategy use by participants and not delayed fraction value construction.

The mathematical model is instantiated as a multilayered dynamic field theory model (e.g., Erlhagen \& Schöner, 2002; Sandamirskaya, 2014) that employs neural tuning curves related to numerical cognition (e.g., Prather, 2012, 2014; 2017) (see Figure 2). Neural tuning curves correspond to patterns of activation in a neural population that is associated with a particular stimulus. For example, numerical stimuli have been found to evoke activity in parietal areas in humans and other species (e.g., Nieder \& Miller, 2005; Piazza, Izard, Pinel, Le Bihan, \& Dehaene, 2004). Activity for a given neuron may be selective for a specific number, meaning that the maximum firing rate is associated with a specific numerical value $\mathrm{N}$, and firing is less for $\mathrm{N}+1, \mathrm{~N}-1$ and so on. This type of coding creates a Gaussian-like neural tuning function. Each number magnitude is not coded exactly, but in a manner that is consistent with Weber-Fechner's law (Fechner, 1966 
FRACTION VALUES

[1860]), which holds that noticeable differences between perceptual stimuli are a function of the proportional difference. As the magnitude of the number increases the neural tuning function width increases proportionally. For example, the width of the tuning function for the magnitude 5 is half that of the magnitude 10 , which is half that of 20 . Thus, differences in the perceived value are a function of the proportional stimulus differences, as with Weber-Fechner's law.

Model Specifications and Procedure. All model instantiations were implemented using MATLAB (Mathworks). We used a dynamic systems model (Beer, 2000; Kelso, 2000). The model architecture was a four-level feed forward neural network with four input layers, two percept layers, and one decision layer. Layers modeled neural tuning curves associated with neural coding of stimuli (e.g., Prather, 2012, 2014; Tudusciuc \& Nieder, 2007). On each trial, the external inputs for the model were the four fraction components that make up the fractions to be compared. The four values were represented by proportionally scaled Gaussian curves that reproduce the classic ratio dependent number distance effect. Comparisons between values that are close and more error-prone and longer to make than comparisons between values that are farther apart. This difference scales with the proportional difference between the values, e.g., 30 vs. 60 is equivalent to 45 vs. 90 . The input layers fed to fraction value layers which converted the numerator and denominator into some value between 0 and 1 . This value was represented using the same Gaussian curve manner.

The internal decision layer connections were specified to produce competition within the layer through lateral inhibition and self-excitation. Thus, the two perceptual 
FRACTION VALUES

layers' output created competition within the decision layer. The "decision" was defined as the location (i.e., index) of the first stable activation peak in the decision layer (the activity with a peak value at the same layer index for ten consecutive time-steps). If the model did not resolve within the allotted time-steps, no decision would be made. The lack of resolution is an issue when there is a large amount of noise in the decision layer. We adjusted the noise to be low enough to avoid a lack of convergence.

Each trial was comprised of 600 time steps. Activation value vectors corresponding to neural tuning curves were calculated using a modified Gaussian distribution function that varies in height similar to a Poisson distribution. The maximum value of the tuning curve $h$, varies with the numerical value $(y)$, such that $h=(121-\mathrm{y})$. The relative width of the calculated curves varied with the value of $S$, which varies across individuals. The mean of the distribution, $m$ is a constant set to 0 . The distance between the target numerical value (T) and the current vector index (V) is defined as $X=\log _{20} T-\log _{20} V$. The method of defining $X$ by logarithmic differences results in Gaussian functions that are symmetric on a log scale and of identical width.

Results and Discussion

Model Condition A. We evaluate how the timing of model decisions across all conditions compared to behavioral data in experiments 1 and 2. Mean response time (in time steps) was 298 for No Delay trials, 334 for Numerator Delay trials and 326 for Denominator delay 
FRACTION VALUES

trials. We also compared double numerator delay $(\mathrm{M}=334)$ to double denominator delay $(M=326)$ and found no significant difference between the two, $t(62)=1.86, p=0.066$.

Model Condition B. We evaluate the number of time-steps taken in completing each comparison. Model completed the same comparison trials as participants. Mean response time (in time steps) was 298 for No Delay trials, 250 for Numerator Delay trials and 324 for Denominator delay trials. We also compared double numerator delay $(\mathrm{M}=250)$ to double denominator delay ( $\mathrm{M}=324$ ) and found numerator delay trials were significantly faster, $t(63)=4.42, p<0.001$.

Model Condition C. For model condition C we investigate how the model would behave if it used a natural number comparison shortcut. We evaluate the number of timesteps taken in completing each comparison. Model completed the same comparison trials as participants. Mean response time (in time steps) was 362 for No Delay trials, 365 for Numerator Delay trials and 356 for Denominator delay trials. We also compared numerator delay trials $(M=365)$ to denominator delay trials $(M=356)$ and found no significant difference between the two, $t(63)=1.45, p=0.15$.

\section{Model Study Conclusions}

We compare three separate model approaches to fraction value construction and comparison. Only Model condition B, in which the denominator initiated fraction value construction, matched participant data from Experiments 1 and 2. Model condition B 
FRACTION VALUES

shows a faster response time for numerator delay conditions, the same is the behavioral data from experiments 1 and 2. Mathematical modeling results suggest that faster response times when numerators are delayed may be because of the fraction value construction process. The mathematical model version in which fraction value construction only requires a denominator to initiate replicates the behavioral data from experiments 1 and 2 . The mathematical model condition A that simply incorporates fraction components as they are available does not match the behavioral data. Model condition A and $\mathrm{C}$ showed no difference in response time for numerator and denominator delay trials.

We interpret the modeling study results as additional evidence that participants construct fraction values in a manner consistent with Model Condition B. Participants can begin the construction of a fraction value once the denominator is available. The availability of a numerator in isolation is no better than no information regarding the fraction at all.

\section{General Discussion}

Using a dynamic stimulus presentation, we reveal a novel behavioral phenomenon in which the fraction comparison response time varied based on the availability of the denominators or numerators. We further demonstrated using a series of mathematical models that the behavioral data are consistent with the hypothesis that people construct fraction values beginning with the denominator value. People may begin to construct the fraction value even when the numerator is unknown. The present hypothesis, described via a mathematical model, is that people's construction of fraction values requires knowledge 
FRACTION VALUES

of the denominator. Modeling results demonstrate how such a fraction construction paradigm leads to the pattern of behavioral data observed in experiments 1 and 2 . Models using a fraction construction paradigm that merely combines fraction components once they are available does not produce similar results to the behavioral data. The behavioral and modeling results provide more comprehensive characterization of the underlying processes in fraction comparison.

If participants begin construction of the fraction value when only presented with the denominator, what are they doing during that time? Consider how the model condition results may differ on numerator delay trials. In numerator delay the participants view both denominators for a specified time period, though no information is given that indicates which fraction will be larger. One possibility is that the fraction value is constructed through the denominator more so than the numerator. Is 10/X more or less information than $X / 10$ ? In some sense, $X / 10$ is because the possible values of $X$ are limited if we assume a proper fraction. If so, then we would expect the effect here to depend on the value of the denominator. The smaller the denominator the fewer possible numerators there are.

To borrow a common educational metaphor of fractions being slices of a pie, then knowledge of the denominator gives the person the number of slices the pie must be cut into, but not the number of slices to be taken. Thus the denominator allows for an estimate of how many parts the whole will be divided into. The size of the pieces (the denominator) is more important information than the number of pieces (the numerator).

Does the present data represent a 'true' measure of fraction comparison? Evidence from other work suggests that behavior in fraction comparison is context dependent. The range of fraction comparisons presented to the participant may constrain behavior. The 
FRACTION VALUES

experiment 1 stimulus set, taken from prior work (Meert, Grégoire, \& Noël, 2009)

contained fraction pairs where either the numerators or denominators were the same, e.g., $5 / 7$ vs. $5 / 10$ or $3 / 7$ vs. $1 / 7$. Stimulus sets that contain fractions with common components may lead to the use of shortcuts that are not employed in other cases (Huber et al., 2014). Thus in experiment 2 the stimulus set used was updated to not include common components. Though the overall patter of results is similar it is still possible that other changes in context may affect the relative response times.

Though the current work provides insight into how people construct fraction values variation in context could affect behavior. Of course, that is the case for fraction comparison as it is with arithmetic tasks in general (Prather \& Alibali, 2009) and any tasks. The computational models presented here can in principle be adjusted to account for individual and context differences. For example, experts may have a stronger representation of fraction values due to greater experience. For those participants, the construction of fraction values may be much faster.

What do the conclusions of this study this suggest about fractions?

The results suggest that participants' processing of fraction values initially relies on the presence of a denominator but not a numerator. When only denominators were present in the delay period fraction comparison response times were relatively fast compared to when only numerators were present. We interpret this effect as suggesting that the participants are able to begin to process both fraction values when presented with the denominator. 
FRACTION VALUES

What does this mean for numerical cognition and cognition in general?

Though the current results are specific to fraction comparison, the results can be situated with other domains involving symbolic number. The framework is that researchers characterize the cognitive processes involved in completing the task by leveraging behavioral variation due to seemingly extraneous stimulus details. Examples of this include the numerical Stroop effect (Besner \& Coltheart, 1979; Foltz, Poltrock, \& Potts, 1984; Henik \& Tzelgov, 1982) and the effect of spacing in arithmetic and Algebra (Landy \& Goldstone, 2005).

There is also a key point here that can be extended to human behavior and cognition in general. Though there were several accounts of the behavioral phenomena in the literature neither created specific predictions of variation in behavior as the task varied. While the accounts may roughly fit with the canonical version of the task that does not mean that the accounts tell us anything about the various processes involved in completing the task. The current account makes specific predictions about how behavior varies (e.g., its timing) and provides quantitative and replicable predictions about behavior. By developing a model of the processes involved in producing the behavior, in completing the task, we have a framework for investigating individual differences in behavior.

\section{References}

Beer, R. D. (2000). Dynamical approaches to cognitive science. Trends in Cognitive Sciences, 4(3), 91-99. http://doi.org/10.1016/S1364-6613(99)01440-0

Besner, \& Coltheart. (1979). Ideographic and alphabetic processing in skilled reading of English. Neuropsychologia, 17(5), 467-472. 
FRACTION VALUES

Braithwaite, D. W., Pyke, A. A., \& Siegler, R. S. (2017). A computational model of fraction arithmetic. Psychological Review, 45. http://doi.org/10.1037/rev0000072

Bright, G., Behr, M., Post, T., \& Wachsmuth, I. (1988). Identifying Fractions on number lines. Journal for Research in Mathematics Education, 19(3), 215-232.

Erlhagen, W., \& Schöner, G. (2002). Dynamic field theory of movement preparation. Psychological Review, 109(3), 545-572. http://doi.org/10.1037//0033295X.109.3.545

Foltz, G. S., Poltrock, S. E., \& Potts, G. R. (1984). Mental comparison of size and magnitude: size congruity effects. Journal of Experimental Psychology. Learning, Memory, and Cognition, 10(3), 442-453. http://doi.org/10.1037/0278-7393.10.3.442

Henik, A., \& Tzelgov, J. (1982). Is three greater than five: The relation between physical and semantic size in comparison tasks. Memory \& Cognition, 10(4), 389-395. http://doi.org/10.3758/BF03202431

Huber, S., Moeller, K., \& Nuerk, H. C. (2014). Adaptive processing of fractions - Evidence from eye-tracking. Acta Psychologica, 148, 37-48. http://doi.org/10.1016/j.actpsy.2013.12.010

Kelso, J. A. S. (2000). Principles of dynamic pattern formation and change for a science of human behavior. In E. (Bergman, L.R. and Cairns, R.B. (Ed.), Developmental Science and the Holistic Approach (pp. 63-83).

Landy, D., \& Goldstone, R. L. (2005). The Alignment of Ordering and Space in Arithmetic Computation. Cognitive Science Society, 437-442. 
FRACTION VALUES

Mack, N. K. (1990). Learning Fractions with Understanding: Building on Informal Knowledge. Journal for Research in Mathematics Education, 21(1), 16. http://doi.org/10.2307/749454

Meert, G., Grégoire, J., \& Noël, M.-P. (2009). Rational numbers: componential versus holistic representation of fractions in a magnitude comparison task. Quarterly Journal of Experimental Psychology, 62(8), 1598-1616.

http://doi.org/10.1080/17470210802511162

Nieder, A., \& Miller, E. K. (2005). Neural Correlates of Numerical Cognition in the Neocortex of nonhuman primates. In Cognition.

Obersteiner, A., \& Tumpek, C. (2016). Measuring fraction comparison strategies with eyetracking. ZDM - Mathematics Education, 48(3), 255-266. http://doi.org/10.1007/s11858-015-0742-z

Piazza, M., Izard, V., Pinel, P., Le Bihan, D., \& Dehaene, S. (2004). Tuning curves for approximate numerosity in the human intraparietal sulcus. Neuron, 44(3), 547-55. http://doi.org/10.1016/j.neuron.2004.10.014

Prather, R. W. (2012). Connecting neural coding to number cognition: a computational account. Developmental Science, 15(4), 589-600. http://doi.org/10.1111/j.14677687.2012.01156.x

Prather, R. W. (2014). Numerical discrimination is mediated by neural coding variation. Cognition, 133(3), 601-610. http://doi.org/10.1016/j.cognition.2014.08.003

Prather, R. W., \& Alibali, M. W. (2009). The development of arithmetic principle knowledge: 
FRACTION VALUES

How do we know what learners know? Developmental Review, 29(4), 221-248. http://doi.org/10.1016/j.dr.2009.09.001

Prather, R. W., \& Heverly-Fitt, S. (2016). Neural coding partially accounts for the relationship between children's number-line estimation and number comparison performance. Frontiers in Developmental Psychology.

Sandamirskaya, Y. (2014). Dynamic neural fields as a step toward cognitive neuromorphic architectures. Frontiers in Neuroscience, 7(January), 1-13. http://doi.org/10.3389/fnins.2013.00276

Simmering, V. R., Schutte, A. R., \& Spencer, J. P. (2008). Generalizing the dynamic field theory of spatial cognition across real and developmental time scales. Brain Research, 1202, 68-86. http://doi.org/10.1016/j.brainres.2007.06.081

Smith, L. B., Thelen, E., Titzer, B., \& Mclin, D. (1999). Knowing in the context of acting: the task dynamics of the A-notB error. Psychological Review, 106, 235-260.

Spencer, J. P., Smith, L. B., \& Thelen, E. (2001). Tests of a dynamic systems account of the Anot-B error: the influence of prior experience on the spatial memory abilities of twoyear-olds. Child Development, 72(5), 1327-46.

Tudusciuc, O., \& Nieder, A. (2007). Neuronal population coding of continuous and discrete quantity in the primate posterior parietal cortex. Neuron, 104(36).

\section{Figure Captions}




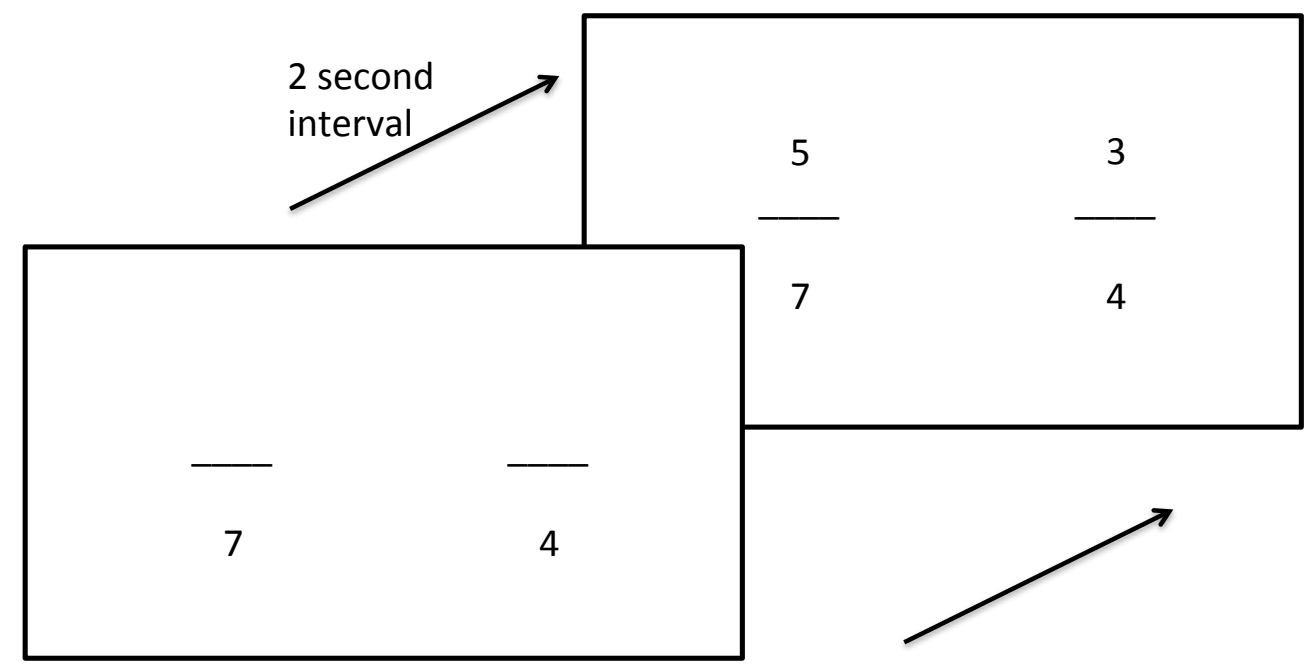

Figure 1. Example of the dynamic fraction display. Two fractions are presented on screen. Depending on the presentation condition a component of the fractions may be blank until the end of the delay period, a two-second interval. Participants are instructed to note which fraction is the larger value via button press. 


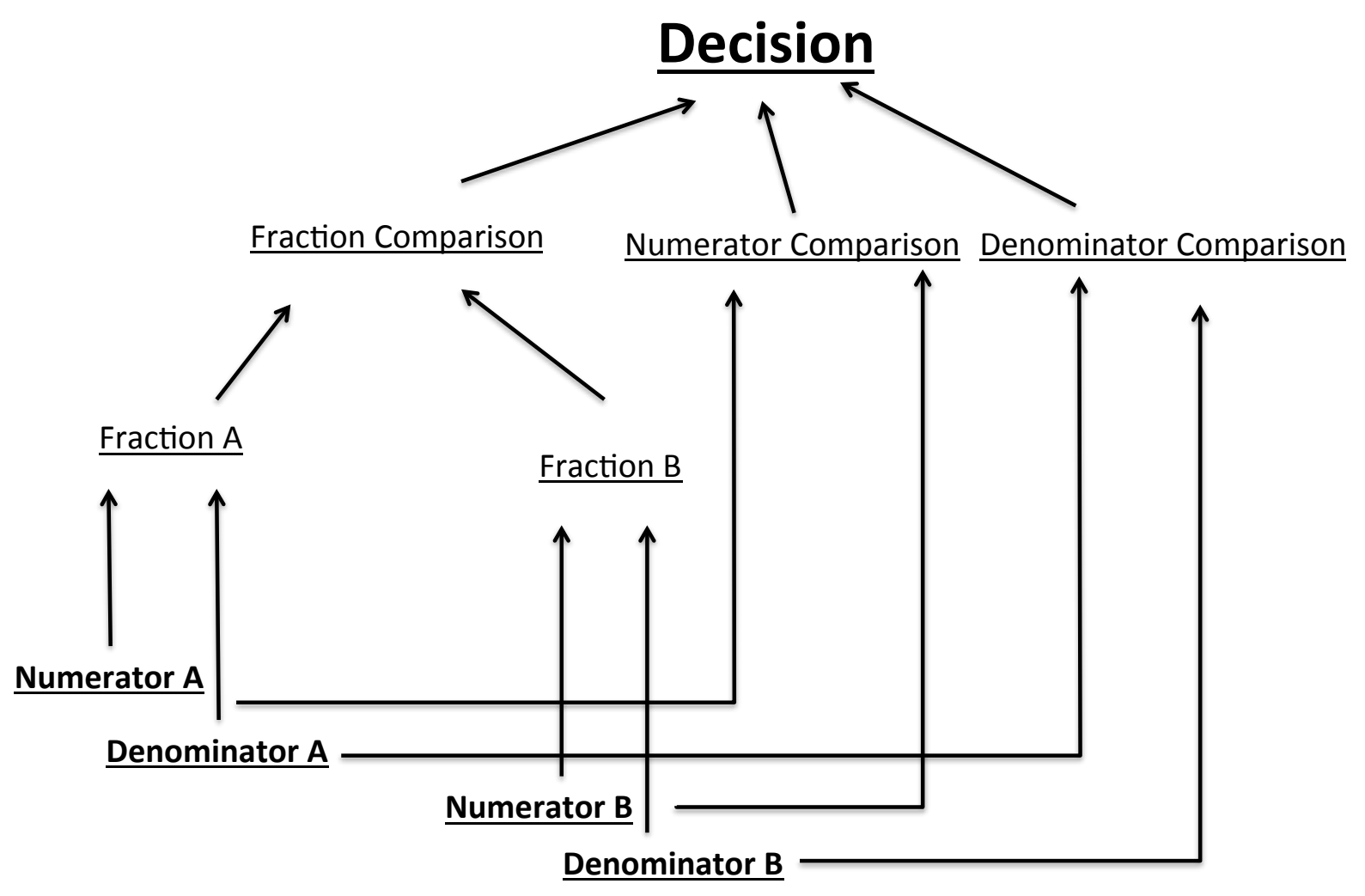

Figure 2. Diagram of the computational model layers and connections. Input layers include Numerator A, Denominator A, Numerator B and Denominator B. The output layer is Decision layer. Arrows correspond to single direction connections of a feed-forward neural network type.

Appendix A 
FRACTION VALUES

\section{Numerator Denominator Numerator Denominator}

A

A

B

B

\begin{tabular}{|c|c|c|}
\hline 3 & 7 & 5 \\
\hline 3 & 8 & 7 \\
\hline 5 & 7 & 4 \\
\hline 7 & 9 & 4 \\
\hline 4 & 9 & 8 \\
\hline 5 & 9 & 7 \\
\hline 8 & 9 & 7 \\
\hline 13 & 14 & 11 \\
\hline 11 & 19 & 17 \\
\hline 12 & 17 & 13 \\
\hline 14 & 17 & 12 \\
\hline 15 & 17 & 12 \\
\hline 12 & 17 & 16 \\
\hline 12 & 19 & 17 \\
\hline 18 & 19 & 12 \\
\hline 14 & 15 & 13 \\
\hline 13 & 16 & 15 \\
\hline 13 & 17 & 16 \\
\hline 17 & 19 & 13 \\
\hline 18 & 19 & 13 \\
\hline
\end{tabular}


FRACTION VALUES

\begin{tabular}{|c|c|c|c|}
\hline 14 & 17 & 15 & 17 \\
\hline 14 & 17 & 16 & 17 \\
\hline 17 & 19 & 14 & 19 \\
\hline 18 & 19 & 14 & 19 \\
\hline 15 & 17 & 16 & 17 \\
\hline 15 & 19 & 17 & 19 \\
\hline 18 & 19 & 15 & 19 \\
\hline 17 & 19 & 16 & 19 \\
\hline 16 & 19 & 18 & 19 \\
\hline 17 & 19 & 18 & 19 \\
\hline 9 & 11 & 3 & 11 \\
\hline 15 & 17 & 11 & 17 \\
\hline 2 & 5 & 2 & 3 \\
\hline 2 & 7 & 2 & 3 \\
\hline 3 & 4 & 3 & 5 \\
\hline 3 & 4 & 3 & 7 \\
\hline 3 & 8 & 3 & 4 \\
\hline 4 & 7 & 4 & 5 \\
\hline 5 & 7 & 5 & 8 \\
\hline 10 & 11 & 10 & 13 \\
\hline 10 & 17 & 10 & 11 \\
\hline 11 & 13 & 11 & 12 \\
\hline
\end{tabular}


FRACTION VALUES

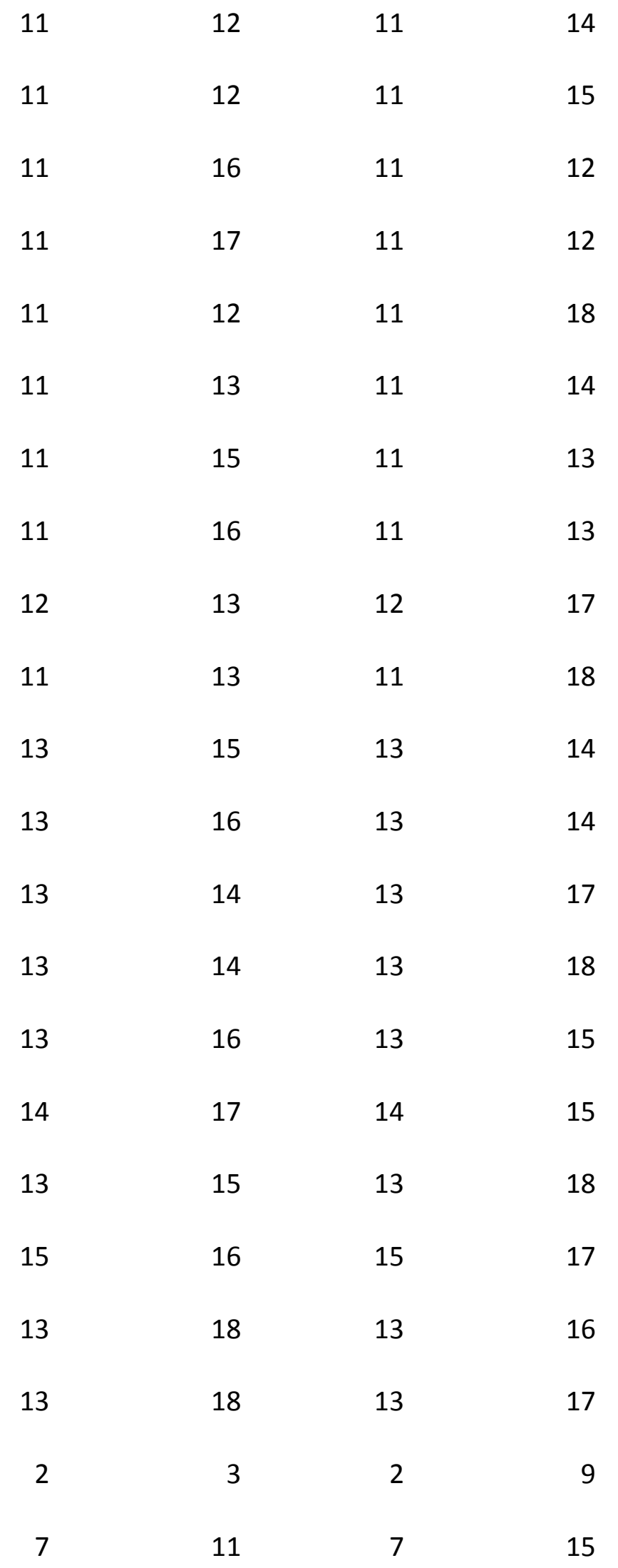


FRACTION VALUES

Appendix B

\begin{tabular}{|c|c|c|c|}
\hline Numerator1 & Denominator1 & Numerator2 & Denominator2 \\
\hline 13 & 19 & 22 & 25 \\
\hline 17 & 21 & 13 & 20 \\
\hline 11 & 23 & 19 & 30 \\
\hline 28 & 43 & 15 & 37 \\
\hline 15 & 31 & 29 & 42 \\
\hline 22 & 35 & 12 & 25 \\
\hline 11 & 18 & 19 & 24 \\
\hline 25 & 48 & 18 & 47 \\
\hline 10 & 17 & 16 & 21 \\
\hline 21 & 29 & 16 & 27 \\
\hline 14 & 31 & 29 & 39 \\
\hline 39 & 44 & 18 & 41 \\
\hline 10 & 23 & 21 & 26 \\
\hline 32 & 39 & 14 & 37 \\
\hline 13 & 32 & 11 & 14 \\
\hline 18 & 23 & 27 & 55 \\
\hline 15 & 26 & 12 & 17 \\
\hline 14 & 29 & 16 & 51 \\
\hline
\end{tabular}


FRACTION VALUES

\begin{tabular}{|c|c|c|}
\hline 21 & 37 & 16 \\
\hline 22 & 27 & 25 \\
\hline 14 & 31 & 11 \\
\hline 21 & 25 & 23 \\
\hline 19 & 39 & 17 \\
\hline 25 & 29 & 30 \\
\hline 22 & 37 & 20 \\
\hline 23 & 26 & 20 \\
\hline 15 & 41 & 13 \\
\hline 10 & 13 & 19 \\
\hline 11 & 28 & 14 \\
\hline 21 & 34 & 12 \\
\hline 16 & 45 & 17 \\
\hline 13 & 18 & 10 \\
\hline 25 & 46 & 15 \\
\hline 26 & 35 & 20 \\
\hline 22 & 31 & 23 \\
\hline 17 & 42 & 11 \\
\hline 26 & 41 & 27 \\
\hline 23 & 36 & 12 \\
\hline 14 & 45 & 19 \\
\hline 24 & 29 & 19 \\
\hline
\end{tabular}


FRACTION VALUES

15

25
38

34

18

37 